\title{
An overlap syndrome of Churg-Strauss syndrome and rheumatoid arthritis
}

\author{
Seung II Bae, Jong Geol Jang, Hun Tae Kim, Hee Yun Ahn, Min Jung Kim, Hyun Je Kim, \\ Choong Ki Lee, Young Hoon Hong \\ Division of Rheumatology, Department of Internal Medicine, College of Medicine, Yeungnam University, Daegu, Korea
}

Churg-Strauss syndrome (CSS) is a necrotizing vasculitis with extra-, peri-vascular eosinophilic infiltration. Chronic symmetric polyarthritis with the presence of rheumatoid factor (RF) and anti-cyclic citrullinated peptide antibody are the mainstay of rheumatoid arthritis (RA) diagnosis. Mononeuritis multiplex is a peripheral neuropathy involving more than 2 separate nerve areas. A 62-year-old male patient was referred for left foot drop and polyarthritis of both hands and feet for 4 months. During evaluation, mononeuritis multiplex was detected on nerve conduction study and electromyography tests: vasculitis with neutrophil, eosinophil, and lymphocyte infiltration on peroneal nerve biopsy. A positive response to methacholin and bronchodilator was observed on the pulmonary function test. Radiologic tests showed peri-articular soft tissue swelling and osteopenia on both hand and foot. Marked peripheral eosinophilia, high RF, and positive perinuclear anti-neutrophil cytoplasmic antibody were detected on blood tests. Here, we report on a patient with overlap syndrome of CSS and RA with review of the relevant literature, from which a few references to overlap syndrome of CSS and RA were available.

Keywords: Churg-Strauss syndrome; Rheumatoid arthritis; Mononeuritis multiplex; Overlap syndrome

\section{INTRODUCTION}

Bronchial asthma, eosinophilia, non-fixed pulmonary infiltrations, para-nasal sinus abnormalities, neuropathies, and extravascular eosinophil infiltration might indicate Churg-Strauss syndrome (CSS). Rheumatoid arthritis (RA) is a prototype of systemic autoimmune inflammatory disease with preferential involvement of joints. Eosinophilia, vasculitis, and arthritis can accompany CSS or RA. However, reports of RA associated with systemic vasculitis are rare, with only a few reported cases involving overlap syndrome of CSS and RA. Here, we report on a patient with overlap syndrome of CSS and RA.

\section{CASE}

A 62-year-old male patient was admitted to our hospital due to progressive left foot drop for 4 months. Symptoms and signs developed insidiously and progressed to an inability to walk by himself, which was associated with pain and motor changes of both hands and feet. Even after conservative treatment, the symptoms and signs had become aggravated. Therefore, the patient was referred from a local medical clinic for further evaluation of peripheral neuropathies without evidence of spinal stenosis or herniated disc on magnetic resonance imaging. The patient was born deaf and verbal communication was impossible, otherwise past medical history and family history were unremarkable, except for intermittent medication for cough and sputum.

At admission, body temperature was $36.7^{\circ} \mathrm{C}$, pulse rate $80 / \mathrm{min}$, respiratory rate $16 / \mathrm{min}$, and blood pressure $140 / 70$ $\mathrm{mmHg}$. The patient complained of excruciating pain and motor weakness of both hands and feet, and showed remarkable swelling, erythema, heat, motion limitations, and tenderness on both wrists, metacarpophalangeal joints and proximal interphalangeal joints (PIPJs) of both hands, both ankles, metatarsophalangeal joints and PIPJs of both feet.

Received: May 12, 2014, Revised: August 19, 2014, Accepted: August 28, 2014

Corresponding Author: Young Hoon Hong, Division of Rheu- matology, Department of Internal Medicine, College of Medicine, Yeungnam University, 170 Hyeonchung-ro, Namgu, Daegu 42415, Korea

Tel: +82-53-620-3820, Fax: +82-53-628-8046, E-mail: yhhongdr@yahoo.co.kr 
In addition to the motion limitation, motor weakness of the feet, graded as G4/G4 on extension, G4/G4 on eversion, G5/G4 on inversion, and G5/G4 on flexion was demonstrated on neurologic examination. However, others were unremarkable on physical examinations and systemic review.

White blood cell (WBC) was 18,250/ $\mu \mathrm{L}$ (neutrophil 39.7\%, lymphocyte $7.5 \%$, eosinophil 49.2\%), hemoglobin $11.0 \mathrm{~g} / \mathrm{dL}$, and platelet $425,000 / \mu \mathrm{L}$ on complete blood count. The measured eosinophil count was $8,979 / \mu \mathrm{L}$ and peripheral blood smear (Fig.1) indicated normochromic normocytic anemia and WBC increased in number with remarkably increased eosinophils with no abnormal cells. Erythrocyte sedimentation rate and C-reactive protein were $54 \mathrm{~mm} / \mathrm{hr}$ and $9.662 \mathrm{mg} / \mathrm{dL}$. Serum lactate dehydrogenase was increased as high as 1,251 $\mathrm{U} / \mathrm{L}$, aspartate transaminase $64 \mathrm{U} / \mathrm{L}$, alanine transaminase 53 $\mathrm{U} / \mathrm{L}$ on liver function test. Serum blood urea nitrogen/creatinine was $12.68 / 0.55(\mathrm{mg} / \mathrm{dL})$. Chest posterioanterior X-ray showed only mildly increased bronchovascular markings on both lung fields.

Evaluations for polyarthritis showed a high rheumatoid factor (RF) of $126.3 \mathrm{IU} / \mathrm{mL}$ ( $<15 \mathrm{IU} / \mathrm{mL}$ ), anti-cyclic citrullinated peptide (CCP) antibody $2.6 \mathrm{IU} / \mathrm{mL}(<10 \mathrm{IU} / \mathrm{mL})$, p-anti-neutrophil cytoplasmic antibody (ANCA) positive, fluorescent antinuclear antibody negative; peri-articular soft tissue swelling and osteopenia on radiological tests (Fig. 2A) and hot uptake of ${ }^{99} \mathrm{mTc}$-methylene diphosphonate of the swollen joints on bone scan (Fig. 2B).

Regarding the motor weakness, nerve conduction study

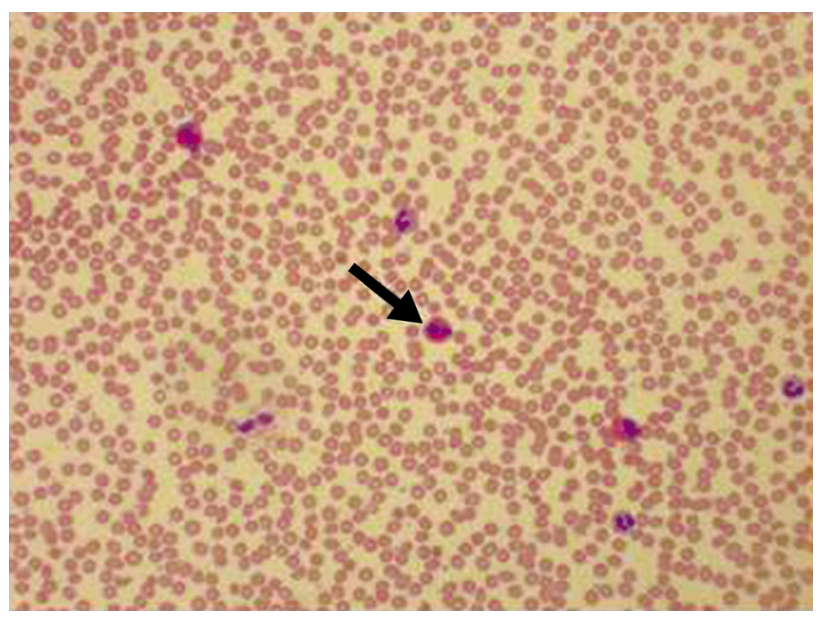

Fig. 1. A peripheral blood smear shows normocytic normochromic anemia and remarkably increased eosinophils (arrow) and white blood cells without abnormal cells (H\&E stain, $\times 40)$.
(NCS) and electromyography (EMG) tests (Fig. 3) demonstrated poor compound motor action potential (CMAP) wave formations, poor F-wave formations, and poor sensory nerve action potential (SNAP) wave formations in the right and left peroneal nerves: Low SNAP amplitudes in the right and left superficial peroneal nerves. Poor F-wave formations and prolonged H-reflex latency in the left posterior tibial nerves. Prolonged F-wave latency in the right posterior tibial nerve. Low CMAP amplitude and motor conduction velocity in the right and left posterior tibial nerve. Prolonged terminal latency, low CMAP amplitude in the left median nerve, low SNAP amplitudes in finger-wrist, wrist-elbow, and palm-wrist segments, low sensory conduction velocities in wrist-elbow and palm-wrist segments of the left median nerve. Prolonged F-wave latency in the right ulnar nerve, prolonged terminal latency, low CMAP amplitude, and low motor conduction velocity above the elbow-axilla segment of the right ulnar nerve. Low SNAP amplitude, low sensory conduction velocities in finger-wrist and wrist-elbow segments of the right ulnar nerve. The results suggested a painful asymmetric asynchro-
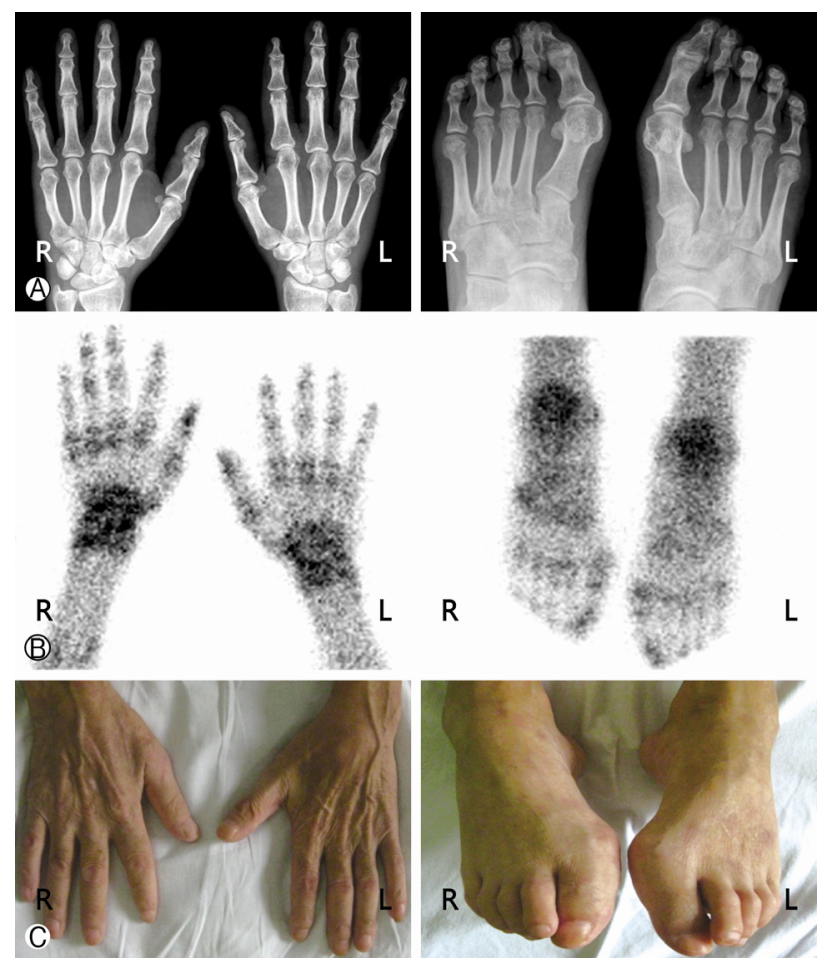

Fig. 2. Soft tissue swelling and peri-articular osteoporosis are found on X-rays (A), and hot uptakes on the proximal interphalangeal joints, metacarpophalangeal joints, both wrists, and ankles are shown on bone scan (B). Photographs of hands and feet (C). 
Motor NCS
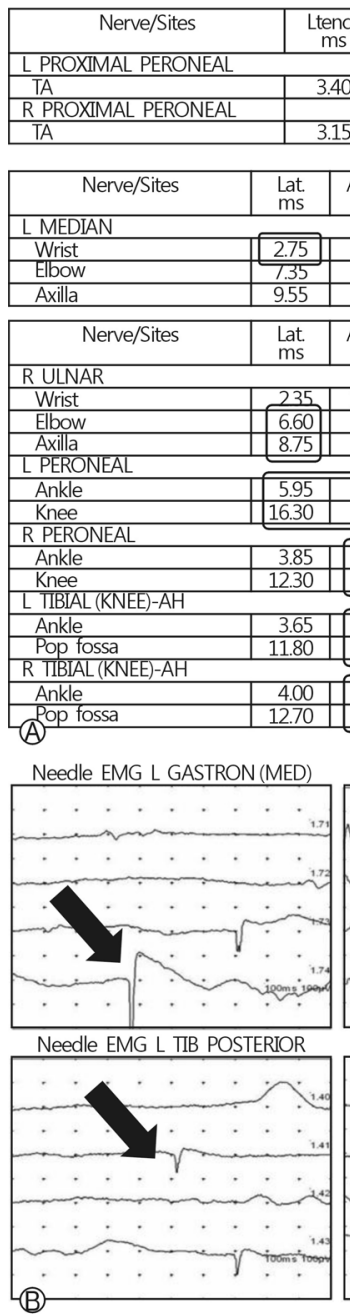

\begin{tabular}{|c|c|c|c|}
$\mathrm{Amp}^{2-3}$ & $\begin{array}{c}\text { Dist } \\
\mathrm{cm}\end{array}$ & $\begin{array}{c}\mathrm{NCV} \\
\mathrm{m} / \mathrm{s}\end{array}$ \\
\hline
\end{tabular}

\begin{tabular}{|c|c|c||c|}
\hline & 3.9 & 11.8 & 34.7 \\
\hline 5 & 2.8 & 12.2 & 38.7 \\
\hline
\end{tabular}

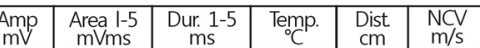
mi mVms

\begin{tabular}{l|l|l|}
\hline 9.6 & 31.2 & 21.55 \\
\hline 8.6 & 29.4 & 22.60 \\
\hline 8.2 & 28.4 & 22.65
\end{tabular}

\begin{tabular}{|l|l|l|l|l|l|}
8.6 & 29.4 & 22.60 & & 25.2 & 54.8 \\
\hline 8.2 & 28.4 & 22.65 & 34.2 & 13.6 & 618 \\
\hline
\end{tabular}

\begin{tabular}{c|c|c|c|c|c|}
$\begin{array}{c}\text { Amp } \\
\mathrm{mV}\end{array}$ & $\begin{array}{c}\text { Area l-5 } \\
\text { mVms }\end{array}$ & $\begin{array}{c}\text { Dur. 1-5 } \\
\mathrm{ms}\end{array}$ & $\begin{array}{c}\text { Temp. } \\
{ }^{\circ} \mathrm{C}\end{array}$ & $\begin{array}{c}\text { Dist. } \\
\mathrm{cm}\end{array}$ & $\begin{array}{c}\mathrm{NCV} \\
\mathrm{m} / \mathrm{s}\end{array}$ \\
\hline
\end{tabular}

$\mathrm{mV}$ mVms

\begin{tabular}{l|l}
\hline .5 & 48.9 \\
\hline 9.8 & 46.0 \\
\hline 9.6 & 43.4
\end{tabular}

46.0

\begin{tabular}{l|l|}
\hline 0 & 25.60 \\
\hline & 26.05 \\
\hline
\end{tabular}

\begin{tabular}{|l|l|l|l|}
\hline 5.60 & & & \\
\hline 6.05 & & 26.2 & 61.6 \\
\hline 320 & & 142 & 660 \\
\hline
\end{tabular}

334
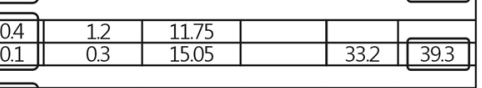

9.60

\begin{tabular}{|l|l|l|l|}
\hline .45 & & & \\
\hline .05 & & 37.7 & 46.3 \\
\hline
\end{tabular}

\begin{tabular}{l||l|l|}
\hline 3.1 & 9.0 & 20.45 \\
\hline 1.2 & 3.6 & 21.05 \\
\hline
\end{tabular}

\begin{tabular}{||c|c|c|c|}
\hline 8.1 & 17.4 & 19.65 & \\
\hline 3.8 & 10.1 & 21.15 &
\end{tabular}
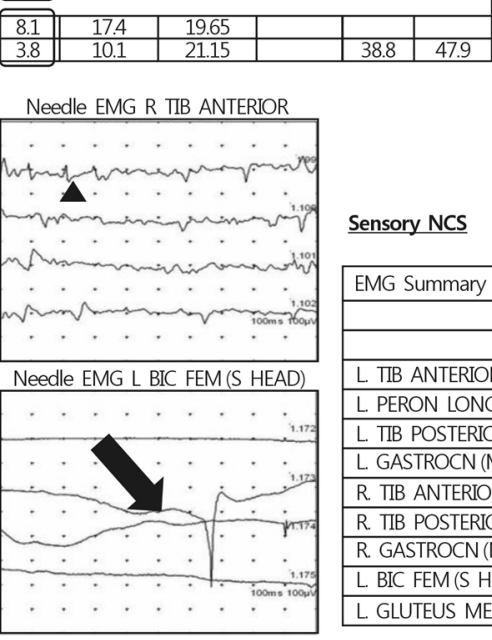

Sensory NCS
Sensory NCS

\begin{tabular}{|l|c|c|c|c|c|c|}
\hline Nerve/Sites & $\begin{array}{c}\text { Lat } \\
\mathrm{ms}\end{array}$ & $\begin{array}{c}\text { Amp }^{2-3} \\
\mathrm{mV}\end{array}$ & $\begin{array}{c}\text { Dur. } \\
\mathrm{ms}\end{array}$ & $\begin{array}{c}\text { Area } \\
\mathrm{mV} \text { ms }\end{array}$ & $\begin{array}{c}\text { Dist. } \\
\mathrm{cm}\end{array}$ & $\begin{array}{c}\mathrm{NCV} \\
\mathrm{m} / \mathrm{s}\end{array}$ \\
\hline SUP PERONEAL-Foot & 2.90 & 4.2 & 1.40 & 2.2 & 14 & 48.3 \\
\hline Rt. side & 3.15 & 2.5 & 1.25 & 1.0 & 14 & 44.4 \\
\hline Lt. side
\end{tabular}

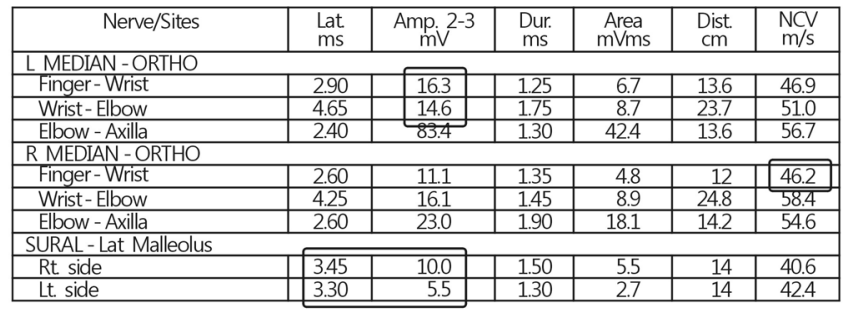

Sensory NCS

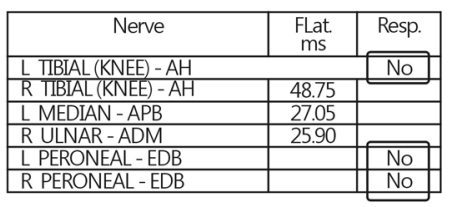

Sensory NCS

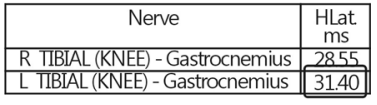

\begin{tabular}{|c|c|c|c|c|c|c|c|c|c|}
\hline \multicolumn{10}{|l|}{ EMG Summary Table } \\
\hline & \multicolumn{5}{|c|}{ Spontaneous } & \multicolumn{3}{|c|}{ MUAP } & Recruitment \\
\hline & LA & Fib & PSW & Fasc & H.F. & Amp & Dur. & PPP & Pattern \\
\hline L TIB ANTERIOR & $\mathrm{I}+$ & $\mathrm{I}+$ & $\mathrm{I}+$ & None & None & $\mathrm{N}$ & $\mathrm{N}$ & $\mathrm{N}$ & Reduced \\
\hline L PERON LONGUS & $\mathrm{N}$ & None & None & None & None & $\mathrm{N}$ & $\mathrm{N}$ & $\mathrm{N}$ & Reduced \\
\hline L TIB POSTERIOR & $\mathrm{I}+$ & I+ & $\mathrm{I}+$ & None & None & & & & \\
\hline L GASTROCN (MED) & I+ & I+ & I+ & None & None & & & & \\
\hline R. TIB ANTERIOR & I+ & I+ & $\mathrm{I}+$ & None & None & $\mathrm{N}$ & $\mathrm{N}$ & $\mathrm{N}$ & Reduced \\
\hline R. TIB POSTERIOR & $\mathrm{N}$ & None & None & None & None & & & & \\
\hline R. GASTROCN (MED) & $\mathrm{N}$ & None & None & None & None & & & & \\
\hline L BIC FEM (S HEAD) & I+ & I+ & $\mathrm{I}+$ & None & None & & & & \\
\hline L GLUTEUS MED & $\mathrm{N}$ & None & None & None & None & & & & \\
\hline
\end{tabular}

Fig. 3. Nerve conduction study shows low compound motor action potential amplitudes and motor conduction velocity in both tibial nerves, low sensory nerve action potential amplitudes in both peroneal nerves (abnormal finding in black square) (A), and electromyography shows increased insertional activities, fibrillations (arrow), positive sharp waves (head arrow) and reduced recruitments in left tibialis posterior, gastrocnemius, biceps femoris, and both tibialis anterior muscles (B).

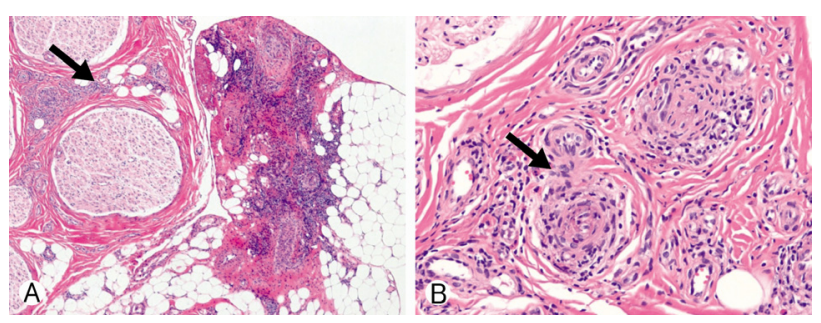

Fig. 4. Microscopic findings of peroneal nerve. Lymphocytes, eosinophils, and neutrophils are infiltrated within and around the walls of small vessels in the epineurium and adjacent soft tissues (arrows). Vascular damage is noted. No pathologic changes are present in the nerve axons and myelin (H\&E stain, $A \times 40$; B $\times 200)$. nous sensory and motor peripheral neuropathy involving isolated damage to multiple separate nerve areas.

Under the impression of mononeuritis multiplex peroneal nerve, a biopsy (Fig. 4) was performed, which detected lymphocyte, eosinophil, and neutrophil infiltrations within and around the walls of small blood vessels present in the epineurium and adjacent soft tissue, consistent with leukocytoclastic vasculitis. However, the axon and myelin showed no pathologic abnormality.

Because peripheral eosinophilia is mainly associated with parasitic infections and allergic disease, a pulmonary function test and methacholin stimulation test in the context of a chro- 


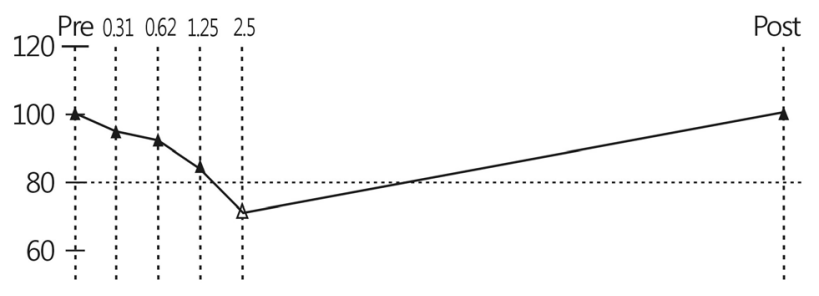

Fig. 5. There is significant reduction of $\mathrm{FEV}_{1}\left(\mathrm{PC}_{20}{ }^{\mathrm{a}}\right)$ of $\mathrm{FEV}_{1}$, 1.59) and response to bronchodilator (more than 20\%) on methacholin stimulation test, which is consistent with bronchial asthma. ${ }^{a}$ PC20: The provocative concentration $(\mathrm{mg} / \mathrm{mL})$ causing a $20 \%$ fall in $\mathrm{FEV}_{1}$.

nic intermittent cough and sputum, as well as stool examinations for parasites were performed. The results showed positive helminth ova for Clonorchis sinensis and small airway obstruction with positive and significant responses to methacholin stimuli and broncho-dilator, consistent with asthma (Fig. 5).

Methylprednisolone $1 \mathrm{~g} /$ day was administered intravenously for 5 days and praziquantel $1,500 \mathrm{mg}$ was prescribed until pain and weakness showed improvement. However, despite complete resolution of swelling of the hands and feet, heat, erythema, passive motion limitations, and tenderness, motor weakness, neuralgia persisted. Follow-up neurologic examinations and NCS/EMG detected no interval changes, even with prednisolone $60 \mathrm{mg} /$ day for 2 months. Peripheral eosinophilia did not disappear, and showed complete fluctuation in number, up to $5,983 / \mu \mathrm{L}$, even though the follow-up stool examinations showed no eggs after praziquantel therapy. While tapering prednisolone down to $10 \mathrm{mg}$ once a day with methotrexate $7.5 \mathrm{mg} 4$ times a week and azathioprine $50 \mathrm{mg}$ twice a day (b.i.d.), gabapentin $100 \mathrm{mg}$ b.i.d. was prescribed along with tramadol $37.5 \mathrm{mg}$ b.i.d. We are following up on the patient without flare-up or exacerbation of disease for one month. The patient was diagnosed as an overlap syndrome of CSS and seropositive RA.

\section{DISCUSSION}

Peripheral eosinophilia is defined by an absolute eosinophil count of more than $600 / \mu \mathrm{L}$ in peripheral blood [1]. Only a few eosinophils circulate in peripheral blood and the majority are present in the gastrointestinal tract, respiratory tract and extra-, especially peri-vascular areas [2]. Eosinophilia is classified to familiar and acquired eosinophilias, and the acquired form is subdivided according to cause into primary and secondary eosinophilias. Primary causes of eosinophilia include clonal diseases, such as acute leukemia and chronic myeloid diseases. Non-infectious causes of eosinophilia include drugs, toxins, allergic reaction and diseases, idiopathic autoimmune inflammatory diseases, malignancies, and endocrine diseases, and infectious causes; parasitic, viral, and bacterial infections [1,3]. Typical vasculitis, such as CSS, Wegener's granulomatosis and polyateritisnodosa, Kimura disease, eosinphilic fasciitis, systemic sclerosis, polyarteritis, other connective tissue diseases, sarcoidosis, and inflammatory bowel diseases are regarded as idiopathic autoimmune inflammatory diseases that can cause eosinophilia.

Mononeuritis multiplex is a painful asymmetric asynchronous sensory and motor peripheral neuropathy involving isolated damage to at least 2 separate nerve areas. Multiple nerves in random areas of the body can be affected. It is associated with or caused by infectious diseases; leprosy, Lyme disease, human immunodeficiency virus, and immune mediated diseases; RA, systemic lupus erythematosus, sarcoidosis, and vasculitis; polyarteritisnodosa, Wegener's granulomatosis, CSS, cryoglobulinemia, amyloidosis, diabetes mellitus, and toxins or drugs.

CSS is a necrotizing vasculitis with extra-, peri-vascular eosinophilic infiltration associated with peripheral eosinophilia, bronchial asthma, or allergic rhinitis clinically. According to the American College of Rheumatology (ACR) criteria, findings of more than 4 from bronchial asthma, eosinophilia (>10\%), non-fixed pulmonary infiltrations, abnormalities of paranasal sinuses, mono- or poly-neuropathies, and extravascular eosinophil infiltration are sufficient for classification as CSS in $85 \%$ sensitivity and $99.7 \%$ specificity [4].

RA is a prototype of systemic autoimmune inflammatory disease with preferential involvement of joints. In addition to chronic inflammatory polyarthritis, extra-articular involvement is also common and critical for RA management. RF and anti-CCP antibody, along with joint involvement, are important for RA diagnosis and disease assessment [5]. In this case, the patient had arthritis in more than 10 joints, RF positive, and increased ESR/CRP. According to 2010 American College of Rheumatology criteria, he met the RA criteria because of the score 8 . Therefore, he was diagnosed with RA.

In an analysis of 214 RA patients by Short et al. [6], 12.4\% of the patients had eosinophilia of more than $4 \%$. However, 
marked peripheral eosinophilia has rarely been reported in RA [7,8]. Panush et al. [7] reported on 5 patients with RA with remarkable eosinophilia ranging between $20 \%$ and $89 \%$, some of which were associated with rheumatoid vasculitis. However, the mechanisms and the significance of eosinophilia in RA remain unclear [9]. Winchester et al. [10] suggested that the prevalence of eosinophilia was higher in RA patients of high RF titer and severe articular or extra articular manifestations than control by $40 \%$.

Although RA can cause eosinophilia, it is critical to rule out other possible diseases for eosinophilia, including malignancies, such as leukemia, infections, and other connective tissue disease [7]. Boudou et al. [11] reported on a patient with chronic eosinophilic pneumonia in RA. Diri et al. [12] reported on a case involving RA with erosive polyarthritis, which developed after remission of CSS. Szilasi et al. [13] reported on four patients with RA with ANCA associated systemic vasculitis including 1 patient with RA with CSS. This patient presented with left foot drop, chronic swelling, and tenderness of both fingers and toes for 4 months, which was sufficient for classification as an overlap syndrome of CSS and RA with mononeuritis multiplex on NCS/EMG tests. The biopsy revealed vasculitis with neutrophil, eosinophil, and lymphocyte infiltration on peroneal nerve. A positive response to methacholin and bronchodilator was observed on the pulmonary function test. Radiologic tests showed periarticular soft tissue swelling and osteopenia on hand, foot; marked peripheral eosinophilia, high RF, positive p-ANCA on blood tests. High dose glucocorticoid was prescribed and was effective for the swollen polyarthritis. However, motor weakness and pain related to the peripheral neuropathy appeared not to be responsive on follow up NCS/EMG tests. Without aggravation of symptoms and signs, we are following up on the patient with $10 \mathrm{mg}$ prednisolone, $100 \mathrm{mg}$ azathioprine, $7.5 \mathrm{mg}$ methotrexate, gabapentin, and tramadol.

In rare cases, RA can include remarkable eosinophilia and vasculitis, and CSS can manifest as arthritis. However, we found a few references to an overlap syndrome of CSS and RA in the English literature. Here we report on a case involv- ing overlap syndrome of CSS and RA with mononeuritis multiplex by vasculitis, bronchial asthma with eosinophilia, and chronic symmetric RF positive polyarthritis.

\section{REFERENCES}

1. Tefferi A. Blood eosinophilia: a new paradigm in disease classification, diagnosis, and treatment. Mayo Clin Proc 2005;80: 75-83.

2. Hochberg MC, Silman AJ, Smolen JS, Weinblatt ME, Weisman MH. Rheumatology. 5th ed. Philadelphia: Elsevier/ Mosby; 2011. p. 183-98.

3. Weller PF, Bubley GJ. The idiopathic hypereosinophilic syndrome. Blood 1994;83:2759-79.

4. Masi AT, Hunder GG, Lie JT, Michel BA, Bloch DA, Arend WP, et al. The American College of Rheumatology 1990 criteria for the classification of Churg-Strauss syndrome (allergic granulomatosis and angiitis). Arthritis Rheum 1990;33:1094100.

5. Turesson C, O'Fallon WM, Crowson CS, Gabriel SE, Matteson EL. Extra-articular disease manifestations in rheumatoid arthritis: incidence trends and risk factors over 46 years. Ann Rheum Dis 2003;62:722-7.

6. Short CL, Bauer W, Reynolds WE. Rheumatoid arthritis. Cambridge, Massachusetts: Harvard University Press; 1957. p. 354-6.

7. Panush RS, Franco AE, Schur PH. Rheumatoid arthritis associated with eosinophilia. Ann Intern Med 1971;75:199-205.

8. Chaudhuri K, Dubey S, Zaphiropoulos G. Idiopathic hypereosinophilic syndrome in a patient with long-standing rheumatoid arthritis: a case report. Rheumatology (Oxford) 2002; 41:349-50.

9. Kargili A, Bavbek N, Kaya A, Koşar A, Karaaslan Y. Eosinophilia in rheumatologic diseases: a prospective study of 1000 cases. Rheumatol Int 2004;24:321-4.

10. Winchester RJ, Koffler D, Litwin SD, Kunkel HG. Observations on the eosinophilia of certain patients with rheumatoid arthritis. Arthritis Rheum 1971;14:650-65.

11. Boudou L, Alexandre C, Thomas T, Pallot-Prade B. Chronic eosinophilic pneumonia (Carrington's disease) and rheumatoid arthritis. Joint Bone Spine 2010;77:477-80.

12. Diri E, Buscemi DM, Nugent KM. Churg-Strauss syndrome: diagnostic difficulties and pathogenesis. Am J Med Sci 2003; 325:101-5.

13. Szilasi M, Mátyus J, File I, Szücs G, Rákóczi E, Pfliegler G, et al. Association of ANCA-associated vasculitis-rheumatoid arthritis overlap syndrome in four patients: rituximab may be the right choice? Autoimmunity 2012;45:304-9. 